\title{
Unobserved heterogeneity in hazard rate models: a test and an illustration from a study of career mobility
}

\author{
HANS-PETER BLOSSFELD ${ }^{(1)} \&$ ALFRED HAMERLE ${ }^{(2)}$ \\ (1) Max-Planck-Institute for Human Development and Education, Lentzeallee 94 , \\ D-100 Berlin 33, Germany (West); ${ }^{(2)}$ Department of Statistics, University of Konstanz, \\ Universitätsstraße 10, D-7750 Konstanz 1, FRG
}

\begin{abstract}
This paper proposes a test for neglected heterogeneity in continuous-time hazard rate models that can be done easily using generally available program packages. It is a score test appropriate for cases in which the variance of the heterogeneity is small and it can be applied quite generally, provided that the generalized residuals can be calculated. The test is demonstrated using data on the career trajectories of German males from the German Life History Study.
\end{abstract}

\section{Introduction}

In recent years, the increasing availability of event history data in biomedical, economics and social science rescarch has led to the widespread application of continuous-time hazard rate models. When using such methods, researchers often assume that they have measured and included the relevant causal influences in the model. But most scientists will agree that in any particular empirical analysis, this is hardly ever the case. Usually some important factors could not be measured or were ignored, creating a spurious change over time in estimated transition rates.

There is a growing literature addressing the issue of omitted variable bias and the problems of how to test and parametrically represent unobserved heterogeneity in continuous-time hazard rate models (cf., for example, Tuma, 1985; Heckman and Singer, 1984; Tuma and Hannan, 1984; Chamberlain, 1985; Trussell and Richards, 1985; Waldman, 1985; Galler and Poetter, 1987; Arminger, 1987). Unfortunately at this point, computer programs designed to alleviate these problems are very specialized and not available to everyone.

The purpose of this paper is, therefore, to propose a test for neglected heterogeneity in continuous-time hazard rate models that can be done easily using generally available statistical program packages, like GLIM (Roger and Peacock, 1983), SAS (1985) or BMDP (Petersen, 1986a, 1986b). We do 
this in three steps. First, we show how unmeasured heterogeneity creates spurious time dependence in observed transition rates. Second, we propose a test procedure which detects this kind of misspecification. Finally, we give an example of the application of this test, analyzing career trajectories of German men from three birth cohorts.

\section{Testing for neglected heterogeneity}

Models for event history data are usually specified in terms of the hazard rate. Let $T$ denote the random duration and let $x$ be the vector of measured covariates. Then the hazard rate is given by

$$
\lambda(t \mid x)=\lim _{\Delta t \rightarrow \infty} \frac{1}{\Delta t} P(t \leqslant T<t+\Delta t \mid T \leqslant t, x) .
$$

Survivor function and density function are

$$
\begin{aligned}
& S(t \mid x)=\exp \left(-\int_{0}^{t} \lambda(s \mid x) \mathrm{d} s\right) \\
& f(t \mid x)=\lambda(t \mid x) S(t \mid x)
\end{aligned}
$$

In (1) it is assumed that the variation in the hazard rate depends only on variation in the measured covariates, i.e, we have assumed that all relevant causal variables have been included in the model. But in many applications there will be variation in the hazard rates that is not adequately measured by the variables in $x$. If such unmeasured heterogeneity is important, then neglecting it will give biased estimates. Let us consider an example. Suppose that a population is divided into two subpopulations $x_{1}$ and $x_{2}$, and that in each subpopulation the hazard is constant, but the two hazard rates are not equal, e.g.

$$
\lambda\left(t \mid x_{1}\right)=0.1, \lambda\left(t \mid x_{2}\right)=0.4 .
$$

Furthermore, let $p_{i}$ be the probability that an individual belongs to subpopulation $i, i=1,2$. The hazard rate in the population is then

$$
\lambda(t)=\frac{\sum_{i} f\left(t \mid x_{i}\right) \cdot p_{i}}{\sum S\left(t \mid x_{i}\right) \cdot p_{i}} .
$$




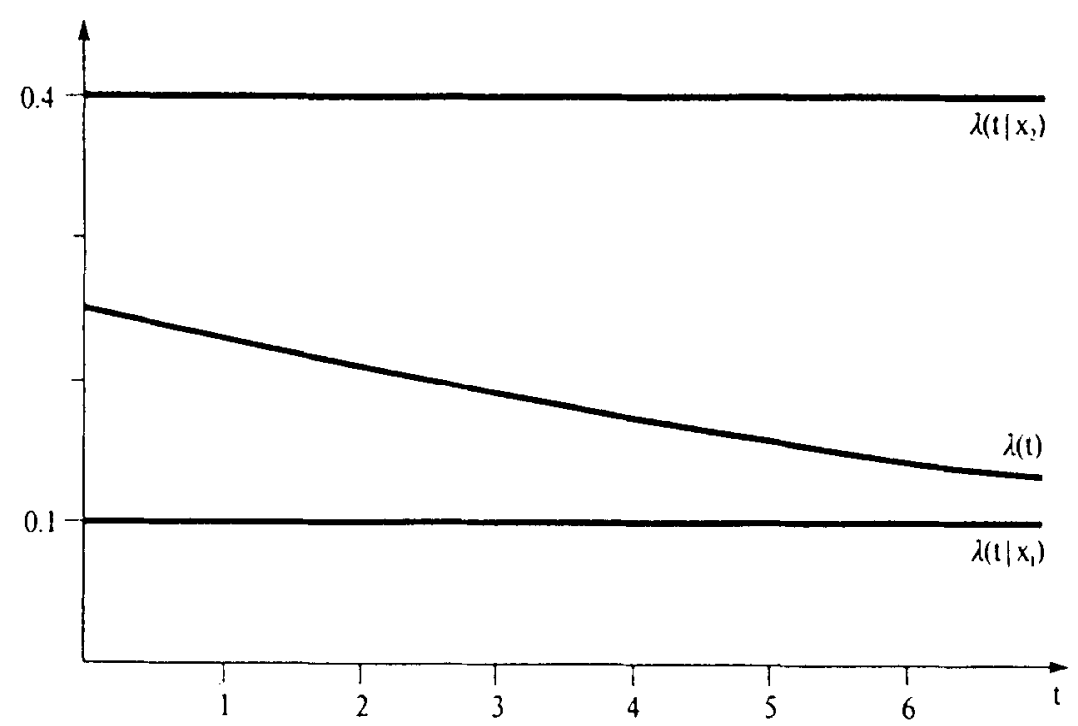

Fig. 1 . The hazard rate path of the subpopulations and the total population $\left(\lambda\left(t \mid x_{1}\right)=0.1\right.$ and $\left.\lambda\left(t \mid x_{2}\right)=0.4\right)$.

For the above values of $\lambda\left(t \mid x_{i}\right)$ and for $p_{1}=p_{2}=0.5$ the hazard rates are given in Figure 1.

Individuals with high rates will experience an event early and persons with high durations will systematically be the individuals with low rates. Therefore the rate in the total population will decline as time elapses. For further examples see Vaupel and Yashin (1985) or Blossfeld, Hamerle, and Mayer $(1986,1988)$. The above mentioned result is general. People will differ along various neglected variables and observed dynamics at the population level will differ from the underlying dynamics at the individual level. In general, failure to control for unobserved variables leads to a bias toward negative duration dependence, i.e., it can lead to an estimated hazard that declines more steeply, or rises more slowly than the true hazard. If the unmeasured heterogeneity is correlated with $x$, then we also expect that neglecting unobserved heterogeneity will result in biased estimates of the regression coefficients. Most of the hazard rate models used in social science to analyze event history data do not account for unobserved heterogeneity, and nearly all available computer programs only estimate models without unobserved heterogeneity. But estimating such a model we cannot be sure if the underlying model is correct or if we have misspecified the model neglecting important unobserved variables. Therefore, we need a test procedure which detects this kind of misspecification, and if possible, we should be able to calculate the test statistic with avialable computer 
programs. In addition, it should not use a special form for the distribution of the heterogeneity.

To include unobserved heterogeneity explicitly in the model, a nonnegative random variable $v$ is defined which has a multiplicative effect on the hazard rate, i.e.,

$$
\lambda(t \mid x, v)=v \cdot \lambda(t \mid x) .
$$

For convenience we assume $E(v)=1$, i.e., "on the average" we have $\lambda(t \mid x)$. The heterogeneity $v$ may vary from individual to individual, but it is time invariant. It is not observable. Furthermore, we asume that $v$ and $x$ are independent. Density and distribution function of $v$ are denoted by $g(v)$ and $G(v)$ respectively.

We define

$$
r(t \mid x)=\int_{0}^{t} \lambda(s \mid x) \mathrm{d} s,
$$

the integrated hazard which is a generalized error term in the sense of Cox and Snell (1968) (see also Crowley and Hu, 1977, or Kalbfleisch and Prentice, 1980), and if we replace unknown parameters in $\lambda$ by maximum likelihood estimates, we have generalized residuals. The survivor function of $r$ is

$$
H(r)=\exp (-r),
$$

which is a unit mean exponential distribution.

For the survivor function of $T$, given $x$ and $v$, we obtain

$$
S(t \mid x)=\exp (-\operatorname{vr}(t \mid x)),
$$

and on integrating this equation with regard to distribution of $v$, we find that

$$
\begin{aligned}
S(t \mid x) & =\int \exp (-\operatorname{vr}(t \mid x)) g(v) \mathrm{d} v \\
& =E_{v}(\exp (-v r(t \mid x)))
\end{aligned}
$$

The density function $f(t \mid x, v)$ is given by

$$
\begin{aligned}
f(t \mid x, v) & =v r^{\prime}(t \mid x) \exp (-v r(t \mid x)) \\
& =v \lambda(t \mid x) \exp (-v r(t \mid x)) .
\end{aligned}
$$


Our test for neglected heterogeneity combines the results of Lancaster (1985), Kiefer (1984), and Burdett et al. (1985). The derivation of the test statistic follows Lancaster. His approach of constructing diagnostics can be applied to any duration model, but he does not consider censored data. We shall generalize his approach to allow for censored durations, but we use the estimator of the test statistic proposed by Burdett et al. Burdett et al. (1985) derive a variance estimator which is conditionally on the estimated parameter values, whereas Lancaster estimates the unconditional variance of the test statistic.

Now we develop the test statistic to examine neglected heterogeneity. The distribution of $v$ is not known, and hence we need a test statistic which does not require knowledge about the form of the distribution of $v$. Let Var $(v)=\sigma^{2}$. The null hypothesis is that there is no neglected heterogeneity. This can be expressed as $\sigma^{2}=0$. With the alternative hypothesis of small $\sigma^{2}$ we approximate the density $f(t \mid x, v)$ and the survivor function $S(t \mid x, v)$ with a Taylor expansion around $v_{0}=1$. Differentiating $S(t \mid x, v)$ with respect to $v$ we obtain

$$
\begin{aligned}
& \frac{\mathrm{d} S(t \mid x, v)}{\mathrm{d} v}=-r(t \mid x) \exp (-v r(t \mid x)) \\
& \frac{\mathrm{d}^{2} S(t \mid x, v)}{\mathrm{d} v^{2}}=r^{2}(t \mid x) \exp (-v r(t \mid x)),
\end{aligned}
$$

and for the Taylor expansions we have

$$
\begin{aligned}
S(t \mid x, v)= & \exp (-r(t \mid x))-r(t \mid x) \exp (-r(t \mid x))(v-1) \\
& +1 / 2 r^{2}(t \mid x) \exp (-r(t \mid x))(v-1)^{2}+R .
\end{aligned}
$$

Integrating $S(t \mid x, v)$ with respect to the distribution of $v$ and neglecting terms of higher order than two, we can write

$$
\begin{aligned}
S(t \mid x) & \approx \exp (-r(t \mid x))+\sigma^{2} / 2 r^{2}(t \mid x) \exp (-r(t \mid x)) \\
& =S(t \mid x, 1)\left(1+\sigma^{2} / 2 r^{2}(t \mid x)\right)
\end{aligned}
$$


For the density $f(t \mid x)$ we obtain

$$
\begin{aligned}
f(t \mid x)= & -\frac{\mathrm{d} S(t \mid x)}{\mathrm{d} t} \approx r^{\prime}(t \mid x) \exp (-r(t \mid x))-\sigma^{2} / 2\left[2 r(t \mid x) r^{\prime}(t \mid x)\right. \\
& \left.\times \exp (-r(t \mid x))+r^{2}(t \mid x) \exp (-r(t \mid x))\left(-r^{\prime}(t \mid x)\right)\right] \\
= & f(t \mid x, 1)-\sigma^{2} r(t \mid x) f(t \mid x)+\sigma^{2} / 2 r^{2}(t \mid x) f(t \mid x, 1) \\
= & f(t \mid x, 1)\left[1+\sigma^{2} / 2 r^{2}(t \mid x)-\sigma^{2} r(t \mid x)\right] .
\end{aligned}
$$

Now we assume that the functional form of $f(t \mid x)$ and $S(t \mid x)$ is known up to a finite dimensional parameter vector $\theta . \theta$ contains the regression coefficients $\beta$ as well as further parameters determining time dependence. We write $f(t \mid x ; \theta)$ and $S(t \mid x ; \theta)$. We introduce a censoring indicator $\delta_{i}$ where $\delta_{i}=0$ if the duration of individual $i$ is right censored and $\delta_{i}=1$ otherwise. The log-likelihood contribution is given by

$$
\log L=\delta \log f(t \mid x ; \theta)+(1-\delta) \log S(t \mid x ; \theta),
$$

where we dropped the subscript $i$ to simplify notation. Using (6) and (7) as an approximation of the true survivor function and the true density function for small $\sigma$ we have

$$
\begin{aligned}
\log L= & \delta\left[\log f(t \mid x, 1 ; \theta)+\log \left(1+\sigma^{2}\left(1 / 2 r^{2}(t \mid x ; \theta)-r(t \mid x ; \theta)\right)\right]\right. \\
& +(1-\delta)\left[\log S(t \mid x, 1 ; \theta)\left(1+\sigma^{2} / 2 r^{2}(t \mid x ; \theta)\right] .\right.
\end{aligned}
$$

The proposed test for neglected heterogeneity is a score test for the hypothesis $\sigma^{2}=0$. Let $\hat{\theta}$ denote the maximum likelihood estimates under the null hypothesis, i.e., when $\sigma^{2}$ is known to be zero. To derive the test statistic we have to calculate the score function at the point $(\hat{\theta}, 0)$. We obtain

$$
\begin{aligned}
\left.\frac{\partial \log L}{\partial \theta_{j}}\right|_{\left(0, \sigma^{2}\right)=(\hat{0}, 0)}=0 \text { for all } j, \\
\begin{aligned}
\frac{\partial \log L}{\partial \sigma^{2}}= & \delta \frac{1 / 2 r^{2}(t \mid x ; \theta)-r(t \mid x ; \theta)}{1+\sigma^{2}\left(1 / 2 r^{2}(t \mid x ; \theta)-r(t \mid x ; \theta)\right)} \\
& +(1-\delta) \frac{r^{2}(t \mid x ; \theta) / 2}{1+\sigma^{2} / 2 r^{2}(t \mid x ; \theta)}
\end{aligned}
\end{aligned}
$$


Evaluating $(\partial \log L) /\left(\partial \sigma^{2}\right)$ at $(\hat{\theta}, 0)$ and collecting terms we see that

$$
\left.\frac{\partial \log L}{\partial \sigma^{2}}\right|_{\left(0, \sigma^{2}\right)=(\hat{0} .0)}=1 / 2 r^{2}(t \mid x ; \hat{\theta})-\delta r(t \mid x ; \hat{\theta}) .
$$

Using the total likelihood function the score test for the null hypothesis $\sigma^{2}=0$ is based on the quantity

$$
S=\frac{1}{2 n} \sum_{i=1}^{n}\left(r^{2}\left(t_{i} \mid x_{i} ; \hat{\theta}\right)-2 \delta_{i} r\left(t_{i} \mid x_{i} ; \hat{\theta}\right)\right) .
$$

The $r\left(t_{i} \mid x_{i} ; \hat{\theta}\right)$ in (9) are the maximum likelihood estimates of the integrated hazards for the model without heterogeneity. Therefore, common computer programs for duration models (e.g., SAS, GLIM, BMDP, RATE) can be used for the computation of $S$.

The variance of $S$ can be estimated by

$$
\operatorname{Va\hat {r}}(S)-\frac{1}{4 n^{2}} \sum_{i=1}^{n}\left(s_{i}-\bar{s}\right)^{2},
$$

where $s_{i}=r^{2}\left(t_{i} \mid x_{i} ; \hat{\theta}\right)-2 \delta_{i} r\left(t_{i} \mid x_{i} ; \hat{\theta}\right)$ and $\bar{s}$ is the sample mean of $s_{i}$. This conditional estimate of the variance of the test statistic was proposed by Burdett et al. (1985), whereas Lancaster (1985) uses an unconditional variance estimator which is more difficult to obtain in the case of censored durations.

The test statistic

$$
\frac{S}{\sqrt{\operatorname{Var}(\bar{S})}}
$$

is asymptotically standard normal, conditionally on the estimated value of $\hat{\theta}$. We use a one-tailed test because the alternative hypothesis is $\sigma^{2}>0$.

The proposed test statistic applies quite general provided that the generalized residuals can be calculated. It is a score test for neglected heterogeneity when the variance of the heterogeneity is small.

\section{An illustration of the test for unobserved heterogeneity}

As an example of the test for unobserved heterogeneity, we analyze the career trajectories of German men from three birth cohorts. In social mobility research, job shift rates are hypothesized to decline as a function 
of time in a job because of job-specific investments in human capital (Mincer 1974). Therefore, a standard continuous-time model for individual career processes would be, any model that allows for a monotonic decrease in the rate of leaving a job (Tuma and Hannan 1984; Blossfeld and Hamerle 1987). In the present case, we use a Weibull model to estimate the job-shift rates of German males in the Federal Republic of Germany. The data are taken from the West German Life History Study (Mayer et al., 1987). This data-base includes information from 2,171 German respondents from the cohorts of 1929-31, 1939-41, and 1949-51 and is representative of the native-born German population of the Federal Republic of Germany (Brückner et al., 1984; Blossfeld, 1987). The data were collected retrospectively by asking the respondents to reconstruct, with exact dates, their life histories. Thus, the data provide the almost complete information about career trajectories, because they tell us the timing of all job moves in sequence. In particular the data-base contains information about the month in which each job held by the respondents started and ended.

In this example, the dependent variable is the number of months a man spent in a job before he moved or censoring occured. The hazard function depends on education (measured in years; cf., Blossfeld and Hamerle, 1987), on prestige of the job (cf., Wegener, 1985), on the number of previous jobs, on the employee's general labor force experience at the time a job was entered (measured in months), and on two dummy variables, indicating the membership of the birth cohort (reference group: cohort 1929-31). In the Weibull model the duration $t$ in a job is used as a proxy for job-specific training.

We assume that there are also unobserved variables, which can be summarized in variable $v$ (omitting subscripts for individual observations), which multiplicatively influence the job-shifts rate of each person. Because hazard rates must be positive, also $v$ must be positive. Since $v$ is unobserved, it is reasonable to assume that $v$ has a mean of unity (Tuma and Hannan, 1984). That is, on average the job-shift rate equals the deterministic function of the included covariates in the Weibull model. The hazard considered is

$$
\lambda(t \mid x, v)=v \alpha t^{\alpha-1} \exp \left(x^{\prime} \beta\right) .
$$

Survivorfunction and probability density are

$$
\begin{aligned}
& S(t \mid x, v)=\exp \left(-v \exp \left(x^{\prime} \beta\right) t^{\alpha}\right), \\
& f(t \mid x, v)=\lambda(t \mid x, v) S(t \mid x, v) .
\end{aligned}
$$

Assume further that $v$ has an exponential distribution, then multiplying $f(t \mid x, v)$ by the probability density of the hazard function $h(v)=\exp (-v)$ 
Table 1. Maximum-likelihood estimates of the effects on the rate of leaving jobs ${ }^{1}$

\begin{tabular}{lcc}
\hline & Model 1 & Model 2 \\
& "Weibull model" & "Log-logistic model" \\
\hline Constant & -3.4240 & -4.3330 \\
& $(0.1433)$ & $(0.1992)$ \\
Duration & 0.8266 & 1.2340 \\
Education & $(0.0129)$ & $(0.0195)$ \\
& 0.0715 & 0.0104 \\
Prestige & $(0.0145)$ & $(0.0209)$ \\
& -0.0049 & 0.0072 \\
Number of previously held jobs & $(0.0014)$ & $(0.0019)$ \\
& 0.1592 & 0.2661 \\
General labor forcc cxpericncc & $(0.0122)$ & $(0.0199)$ \\
& -0.0086 & -0.0126 \\
Cohort 1939-41 & $(0.0005)$ & $(0.0006)$ \\
& 0.1218 & 0.1244 \\
Cohort 1949-51 & $(0.0485)$ & $(0.0726)$ \\
& 0.3325 & 0.3244 \\
-2 log $L$ & $(0.0520)$ & $(0.0803)$ \\
Test statistic for & 5632 & 3294 \\
unobserved heterogeneity & 5.10 & 2.60
\end{tabular}

${ }^{1}$ Standard errors in parenthesis.

and integrating over all possible values of $v$ gives the density of the observed job-shifts rate that is not dependent on the disturbance

$$
\begin{aligned}
f(t \mid x) & =\alpha t^{\alpha-1} \exp \left(x^{\prime} \beta\right) \int_{0}^{\infty} v \exp \left(-v\left(1+\exp \left(x^{\prime} \beta\right) t^{\alpha}\right) \mathrm{d} v\right. \\
& =\frac{\alpha t^{\alpha-1} \exp \left(x^{\prime} \beta\right)}{\left(1+\exp \left(x^{\prime} \beta\right) t^{\alpha}\right)^{2}}
\end{aligned}
$$

Notice that the observed job-shift rate has a log-logistic distribution even though each individual's job-shift rate is assumed to be Weibull distributed. Therefore, if there is standard exponentially distributed unobserved heterogeneity in the job-shift model, our proposed test for unobserved heterogeneity should give a significant result for the Weibull model and a nonsignificant result for the log-logistic model.

The parameter estimates for these two models are obtained by means of the algorithm described by Roger and Peacock (1983), using GLIM. The estimates are reported in Table 1. 
Model 1 in Table 1 assumes that the job-shift rate is Weibull distributed. The estimates show that education and the number of previous jobs had a significant and positive effect on the rate of leaving jobs. With increasing education and increasing number of jobs, people are more mobile. Also significant and positive are the effects of the cohort dummies. Each younger cohort shows more mobility than each older one. Significant and negative is the effect of general labor force experience and prestige. That is, the longer the experience and the higher the prestige of the job, the less people move. The duration dependence is also negative, indicating that the longer a person has been in a job the less likely it is that the person will change, presumably because of job-specific investments in human capital. This finding is in accordance with human capital theory (Mincer, 1974).

Using our proposed score test, we examine the null hypothesis that there is no neglected heterogeneity in our job-shift model. Estimates of the cumulative hazards of the given Weibull model are easily obtained with SPSS (Blossfeld, Hamerle, and Mayer, 1986, 1988). The asymptotically $N(0 ; 1)$ distributed test statistic is highly significant $(z=5.11)$, indicating that there is unobserved heterogeneity in our job-shift model which may lead to spurious findings.

Model 2 assumes, therefore, that the job-shift rate has a Weibull distribution for any job-person unit, but has a log-logistic distribution with the population, due to an unobserved standard exponentially distributed random term for each person. As Table 1 shows, all estimates for the observed covariates have the same sign as in the Weibull model and show the same pattern of statistical significances. The duration dependence is positive, indicating that the rate in the population has a non-monotonic shape even when each individual's rate is assumed to be declining. This may be seen as an example of the general finding described above, namely, that unobserved heterogeneity creates spurious time dependence in observed transition rates.

However, when the score test for the log-logistic model is again calculated, the test statistic still shows a significant result - although much lower than in the Weibull model $(z=2.60)$. In other words, there is still unobserved heterogeneity in our job-shift model. Only a part of the originally neglected heterogeneity could be controlled for by a standard exponential distribution.

This leads us to the question of how to specify the disturbance's distribution in continuous-time hazard rate models. Unfortunately in most cases there is not theory or prior research that can provide a convincing rationale for specifying any particular distribution of the disturbance (Tuma and Hannan, 1984). Morover, Heckman and Singer (1984) as well as Galler and 
Poetter (1987), who estimated several models with different assumptions about the distribution of the disturbance, conclude with warnings not to make casual decisions about distributions of the disturbance. We simply need more research on this topic. Nevertheless, given a specific transition rate model to be used in empirical research, our proposed test can at least show whether there is unobserved heterogeneity and whether the estimations are affected by omitted influences. The test statistic, therefore, provides a useful instrument for model evaluation.

\section{Conclusion}

The present paper has shown how unmeasured heterogeneity can create spurious time dependence in observed transition rates. Because it is often the case in empirical research that important factors cannot not be measured or are ignored, it is very likely that models are misspecified. Unfortunately, to date unobserved heterogeneity could only be estimated using very specialized computer programs, not available to everyone. The purpose of the present paper was, therefore, to propose a test for neglected heterogeneity in continuous-time hazard rate models that can be done easily using generally available program packages.

The proposed test for unobserved heterogeneity combines the results of Lancaster (1985), Kiefer (1984), and Burdett et al. (1985). It is a score test appropriate for cases in which the variance of the heterogeneity is small and it can be applied quite generally, provided that the generalized residuals can be calculated.

The test was demonstrated using data on the career trajectories of German males from the German Life History Study. Using a Weibull model, the test for unobserved heterogeneity showed a highly significant result, indicating that there is unobserved heterogeneity. Assuming that this unobserved heterogeneity would have a standard exponential distribution, leading to a log-logistic distribution within the population, we used the test for unobserved heterogeneity again to examine the loglogistic distribution. The test statistic again showed a significant result - although much lower than for the Weibull model. Thus, only a part of the originally neglected heterogeneity could be controlled for by a standard exponential distributed random variable. Although further research is required to investigate how to represent parametrically unobserved heterogeneity in continuous-time hazard rate models, our proposed test for unobserved heterogeneity nevertheless provides a useful first step in evaluating models. 


\section{Acknowledgments}

This paper results from work undertaken as part of the West German life-history project, which is based in the Max-Planck-Institute for Human Development and Education, and supported by grants of the Deutsche Forschungsgemeinschaft. For helpful comments on an earlier draft, we wish to thank Ellen Skinner and Ulrich Poetter.

\section{References}

Arminger, Gerhard. (1987). "Testing against misspecification in parametric rate models", pp. 679-699 in K.U. Mayer \& N.B. Tuma (eds), Applications of Event History Analysis in Life Course Research. Berlin: Max-Planck-Institut für Bildungsforschung.

Blossfeld, Hans-Peter. (1987). "Zur Repräsentativität der Sfb-3-Lebensverlaufsstudie. Ein Vergleich mit Daten aus der amtlichen Statistik", pp. 126-144 in Allgemeines Statistisches Archiv, 71.

Blossfeld, Hans-Peter \& Alfred Hamerle. (1987). "Interpreting career mobility as a multiepisode process". Working paper no. 236 of the Sonderforschungsbereichs 3, Frankfurt a.M./Mannheim.

Blossfeld, Hans-Peter, Alfred Hamerle \& Karl Ulrich Mayer. (1986). Ereignisanalyse. Statistische Theorie und Anwendung in den Wirtschafts- und Sozialwissenschaften. Frankfurt a.M./New York: Campus.

Blossfeld, Hans-Peter, Alfred Hamerle \& Karl Ulrich Mayer. (1988). "Event history analysis - Statistical theory and application in the social sciences" (Forthcoming: Lawrence Erlbaum, Hillsdale, NJ 1988/89).

Brückner, Erika, H.-P. Kirschner, M. Wiedenbeck \& J. Hoffmeyer-Zlotnik. (1984). Methodenbericht "Lebensverläufe". Mannheim: ZUMA.

Burdett, K., N.M. Kiefer \& S. Sharma. (1985). "Layoffs and duration dependence in a model of turnover", Journal of Econometrics 28: 51-69.

Chamberlain, Gary. (1985). "Heterogeneity, omitted variable bias, and duration dependence", pp. 3-38 in James J. Heckman \& Burton Singer (cds), Longitudinal Analysis of Labor Market Data. Cambridge, Mass: Cambridge University Press.

Cox, D.R. \& E.J. Snell. (1968). "A general definition of residuals", Journal of the Royal Statistical Society, Ser. B, 30: 248-275.

Crowley, John \& Marie Hu. (1977). "Covariance analysis of heart transplant survival data", Journal of the American Statistical Association 72: 27-36.

Galler, Heinz \& Ulrich Poetter. (1987). "Unobserved heterogeneity in models of unemployment duration", pp. 628-650 in K.U. Mayer \& N.B. Tuma (eds), Applications of Event History Analysis in Life Course Research. Berlin: Max-Planck-Institut Für Bildungsforschung.

Heckman, James J. \& Burton Singer. (1984). "A method for minimizing the impact of distributional assumptions in economic models for duration data", Econometrica 52: 63-132.

Kalbfleisch, John D. \& Ross. L. Prentice. (1980). The Statistical Analysis of Failure Time Data. New York: Wiley.

Kiefer, N. (1984). "A simple test for heterogeneity in exponential models of duration", Journal of Labour Economics 2: 539549. 
Lancaster, T. (1985). "Generalized residuals and heterogeneous duration models with applications to the Weibull model", Journal of Econometrics 2: 155-169.

Mayer, Karl Ulrich et al. (1987). Lebensverläufe and Wohlfahrtsentwicklung. Materialien zu Konzeption, Design and Methodik der Hauptuntersuchung 1981/82. Max-Planck-Institut für Bildungsforschung, Berlin.

Mincer, J. (1974). Schooling, Experience, and Earnings. New York: Columbia University Press.

Peterson, Trond. (1986a). "Estimating fully parametric hazard rate models with timedependent covariates", Sociological Methods and Research 14: 219-246.

Peterson, Trond. (1986b). "Fitting parametric survival models with time dependent covariates", The Journal of the Royal Statistical Society, Ser. C, 35: 281-288.

Roger, J.H. \& S.D. Peacock. (1983). "Fitting the scale as a FGLIM parameter for Weibull, extreme value, logistic and log-logistic regression models with censored data", GLIMNewsletter 6: 30-37.

SAS User's Guide: Statistics, Version 5 Edition. (1985). Cary, North Carolina, pp. 507-557.

Trussel, J. \& T. Richards. (1985). "Correcting for unmeasured heterogeneity in hazard models using the Heckman-Singer procedure", in N. Tuma (ed), Sociological Methodology. San Francisco: Jossey-Bass.

Tuma, Nancy B. (1985). "Effects of labor market structure on job-shift patterns", pp. 327-363 in James J. Heckman \& Burton Singer (eds), Longitudinal Analysis of Labor Market Data. Cambridge, Mass: Cambridge University Press.

Tuma, Nancy B. \& Michael T. Hannan. (1984). Social Dynamics: Models and Methods. New York: Academic Press.

Vaupel, J.W. \& A.I. Yashin. (1985). "The deviant dynamics of death in heterogeneous populations", pp. 179-211 in N. Tuma (ed), Sociological Methodology, San Francisco: Jossey-Bass.

Waldman, D.M. (1985). "Computation in duration models with heterogeneity", Journal of Econometrics 28: 127-134.

Wegner, Bernd. (1985). “Gibt es Sozialprestige?” Zeitschrift Für Soziologie 14: 209-235. 